\title{
EXPERIMENTAL STUDY FOR THE IDENTIFICATION OF A TENSORIAL SCHEME FOR SHAPE MEM- ORY ALLOYS
}

\author{
P.Y. MANACH and D. FAVIER \\ Laboratoire Génie Physique et Mécanique des Matériaux (GMP2), U.R.A. CNRS 793, E.N.S.P.G., \\ 101 rue de la Physique, D.U. BP 46, F-38402 St Martin d'Hères cedex, France
}

\begin{abstract}
The industrial development of shape memory alloys needs an accurate prediction of their behavior for using it in C.A.D. programs. The carrying out of such programs needs the definition of reliable thermomechanical constitutive laws. These laws are three-dimensionnal and therefore it is necessary, in order to perform an accurate identification, to do more than only tensile tests. In that way, this study presents some experimental results on the behavior of $\mathrm{Cu}-\mathrm{Zn}-$ Al alloys under two different types of solicitations: uniaxial tension and simple shear. A first comparison between these two tests, in terms of stress equivalent, is also presented. The identification of the shear test results is performed using an elastohysteresis model.
\end{abstract}

\section{I) INTRODUCTION}

The non-classical behavior of shape memory alloys presents several problems for the definition of thermomechanical constitutive laws, such as :

- the mechanical behavior is complex, non linear at constant temperature and dependent on it

- there is a mechanical and a thermal hysteresis

- there is an influence of microstructural parameters (texture, grain size)

- there is a dependence of the thermomechanical history.

In the three-dimensionnal case, the identification of such tensorial laws cannot be achieved using only usual tensile tests; in that way, both homogeneous tensile and shear tests are complementary. Indeed, on one hand, with uniaxial tensile tests, the stress state involves only one diagonal component of the stress tensor; on the other hand, non diagonal components of stress and strain tensors principally appear in the shear tests. Futhermore, materials can be studied using the same form (sheet samples) and both experiments allow the testing of planar anisotropy. Finally, cyclic tests can be easily performed with shear tests.

The shape memory alloy behavior is highly temperature dependent and therefore it is necessary, in order to obtain an accurate and reliable identification, to control the temperature during the tests. $A$ particular attention must be paid to : 
- the mechanical homogeneity of the tests

- the thermal homogeneity of the tests

- an accurate measurement of the homogeneous deformation

\section{II) EXPERIMENTAL DEVICES AND CONDITIONS}

The experimental study was carried out on two types of mechanical tests, such as uniaxial tension and simple shear. These tests were performed at room temperature using a Jacottet hydraulic machine with a maximum capacity of 5000 daN. This study is a first step to test our experimental devices and the reliability of the results.

\section{a) Materials}

The studied materials are copper-zinc-aluminium shape memory alloys, supplied by the Insa of Lyon. At room temperature $\left(20^{\circ} \mathrm{C}\right)$ the alloy $\mathrm{M}$ is martensitic and the alloy $A$ is austenitic. The chemical compositions of these alloys are:

\begin{tabular}{|c|c|c|c|}
\hline Alloy & Copper (\%wt) & Zinc (\%wt) & Aluminium (\%wt) \\
\hline Martensitic M & 70.9 & 25.1 & 4 \\
\hline Austenitic A & 68.8 & 27.2 & 4 \\
\hline
\end{tabular}

A differential scanning calorimetry on each alloy gave the following transformation temperatures :

\begin{tabular}{|c|c|c|c|c|}
\hline Alloy & Ms $\left({ }^{\circ} \mathrm{C}\right)$ & Mf $\left({ }^{\circ} \mathrm{C}\right)$ & As $\left({ }^{\circ} \mathrm{C}\right)$ & Af $\left({ }^{\circ} \mathrm{C}\right)$ \\
\hline Martensitic M & 80 & 60 & 85 & 105 \\
\hline Austenitic A & 6 & -12 & 6 & 14 \\
\hline
\end{tabular}

\section{b) The tensile device}

The tensile test specimens were bone-shaped with a gauge length of 25 $\mathrm{mm}$; the strain rate was $10^{-3} \mathrm{~s}^{-1}$. The deformation was measured with a Shepic extensometer (type SE-0.5E). The accuracy of the measure on the axial deformation $\varepsilon$ is of the order of $10^{-4}$.

\section{c) The simple shear device}

The shearing tests were performed on a shearing machine from C.G'Sell, adapted for metals by E.Rauch [1]. The deformation is measured with a video system, using a CCD camera, which records the inclination of the limit between black and white ink layers (Fig.1).
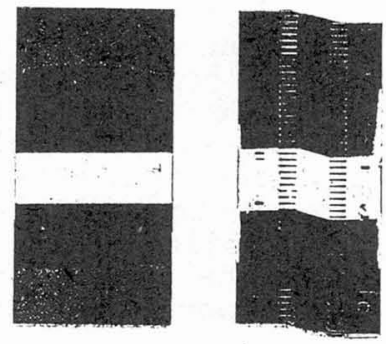

Fig.1 : Cu-Zn-Al samples before and after simple shear test.

The accuracy of this measure on the shear deformation $\gamma$ is of the order of $2.10^{-5}$. 


\section{III) EXPERIMENTAL RESULTS}

\section{a) Reproducibility of the results}

A first step was to check the reproducibility of the tensile and shear tests by loading and unloading the specimen with an increasing deformation. We observe on Fig.2 a good reproducibility. The difference between the curves is less than $5 \%$.
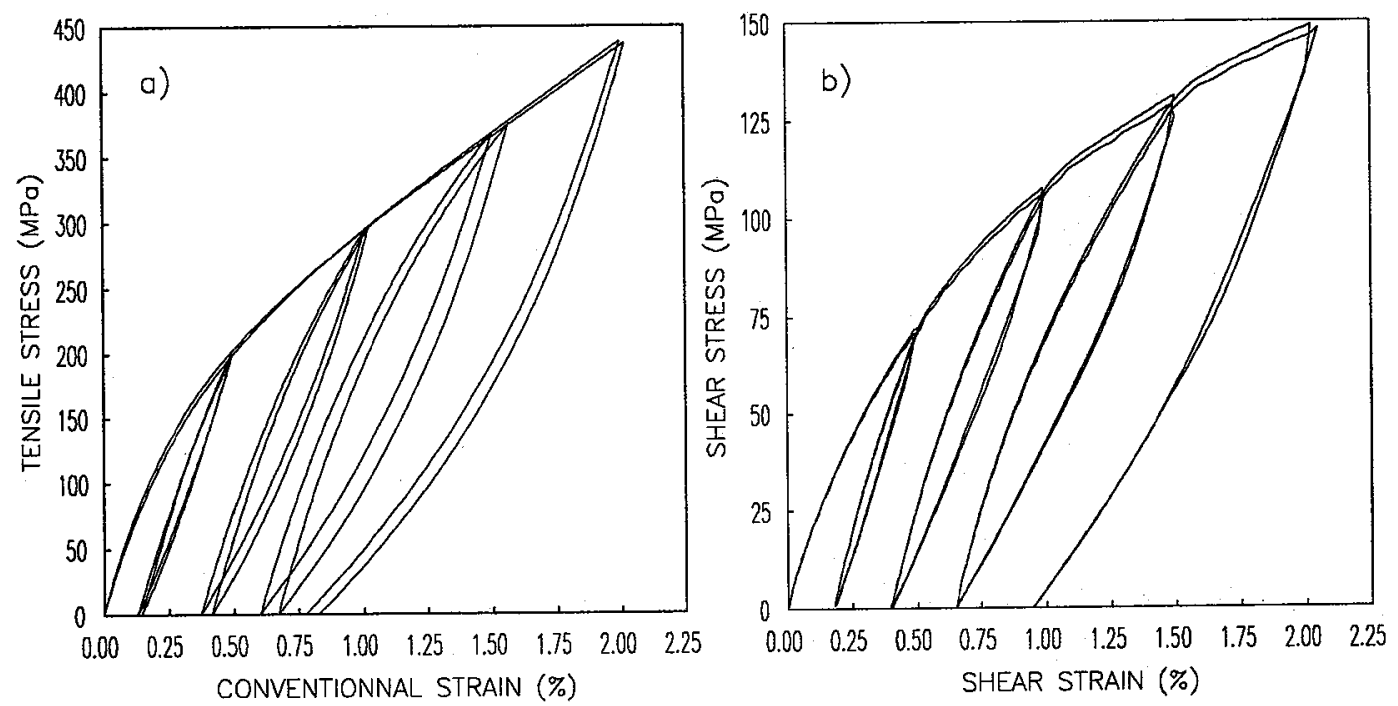

Fig.2 : reproducibility of tension (a) and shear (b) tests in the martensitic phase.

\section{b) Anisotropy}
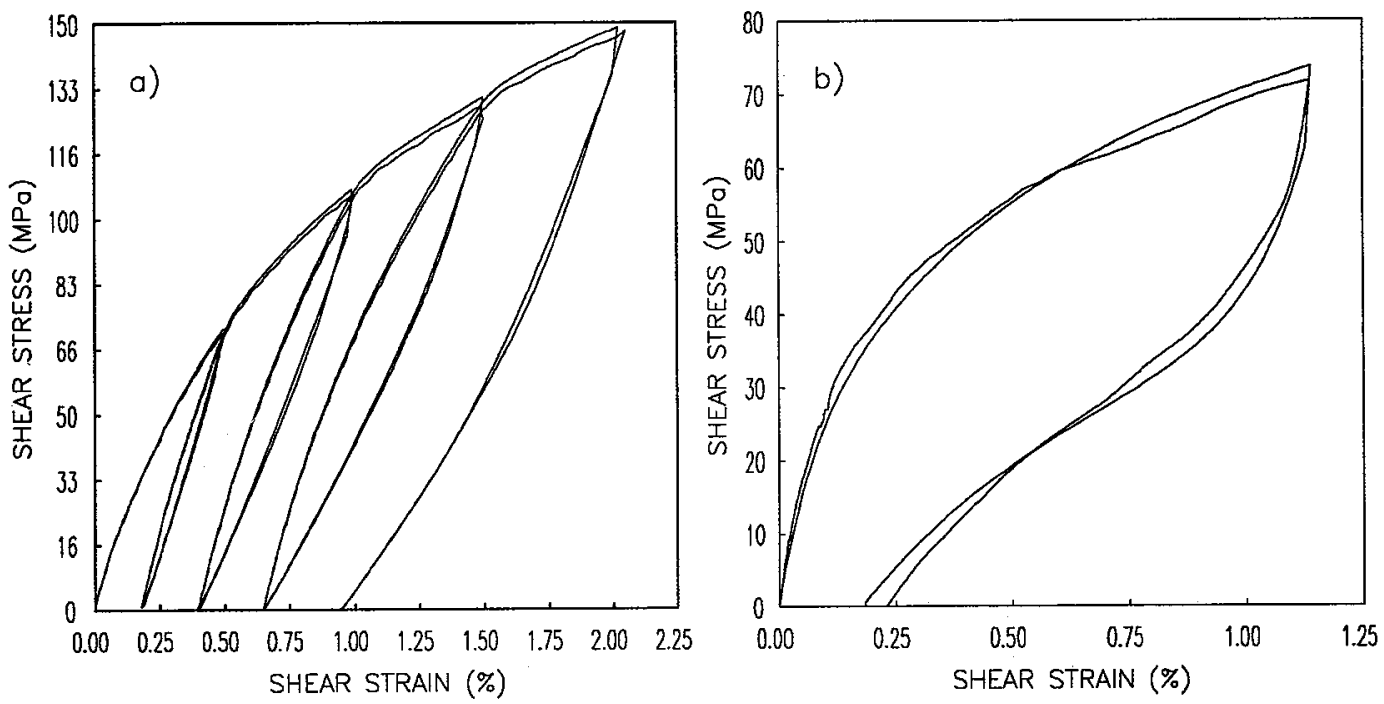

Fig.3: shear tests of 2 different orientations (45० from each other) in the martensitic phase (a) \& in the austenitic phase (b). 
In order to caracterize the anisotropy in the sheet plane, two orientations at $45^{\circ}$ from each other were tested by shearing. The results obtained on the alloys $M$ and $A$ (Fig.3) show no significant anisotropy both in the martensitic and the austenitic phase.

The assumption of planar isotropy (revolution orthotropy) fits well our materials.

\section{c) Comparison between shear and tensile tests}

Experimental results from both tests can be compared in terms of stress equivalents. The equivalence relation between tension and shear is taken as :

\section{$\tau_{\text {shear . Equivalent. } \sigma_{\text {tension }} / \mathrm{K}}$}

Moreover, as far as the main deformation mechanisms are related to the martensitic transformation or to the reorientation of the martensite, we can assume that the deformation is isovolume. As the material is assumed isotropic, the equivalence between deformations can be also written :

\section{$\gamma_{\text {shear }}$.Equivalent. $\varepsilon_{\text {tension }} \cdot \mathrm{K}$}

In order to check these equivalences, shear tests results are plotted in the axes $\tau, \gamma$, and tensile ones in the axes $\sigma / K, \varepsilon . K$ (Fig.4).
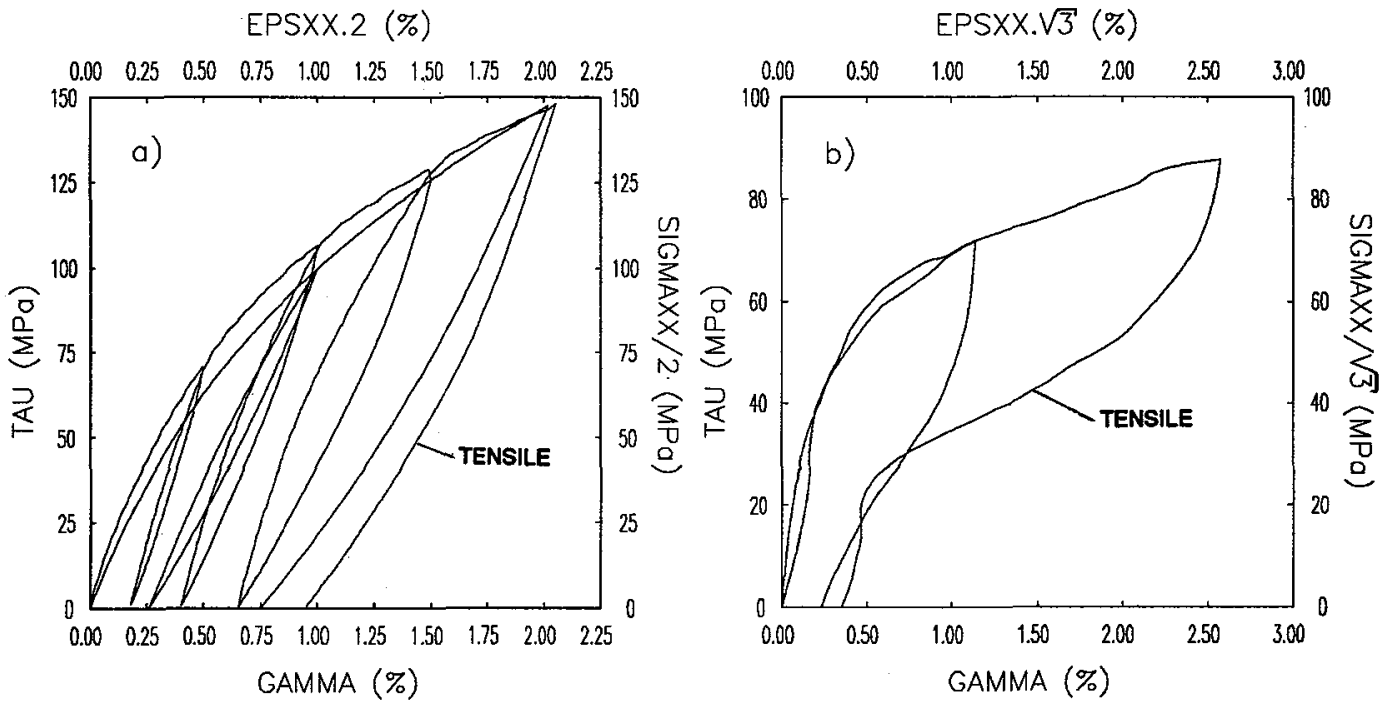

Fig.4 : Comparison between tensile \& shear tests in the martensitic phase (a) \& the austenitic phase (b).

Fig.4.a) shows for the alloy $M$ a comparison between both solicitations with $\mathrm{K}=2$ (Tresca criterion). For the alloy $\mathrm{A}$, it is the Von Mises criterion ( $K=\sqrt{3}$ ) which gives the best result. In fact, comparison using equivalent stress and strain are not sufficient to modelize all the behaviors; the previous results show good agreement, in terms of final stress levels but not in terms of initial and unloading slopes. 


\section{d) Cyclic shearing test}

It is impossible to perform centred cyclic tests in tension on sheet specimen because of buckling. But, such tests are necessary to determine the type of hardening either isotropic or kinematic. These tests were performed by shearing on the alloy $M$. Cycling is done between $+/-1 \%$ of deformation $\gamma$ for 10 cycles followed by 10 cycles at 2\%. We observe (Fig.5) a cyclic hardening, i.e, an increase of the final stress which tends to saturate after 5 or 6 cycles.

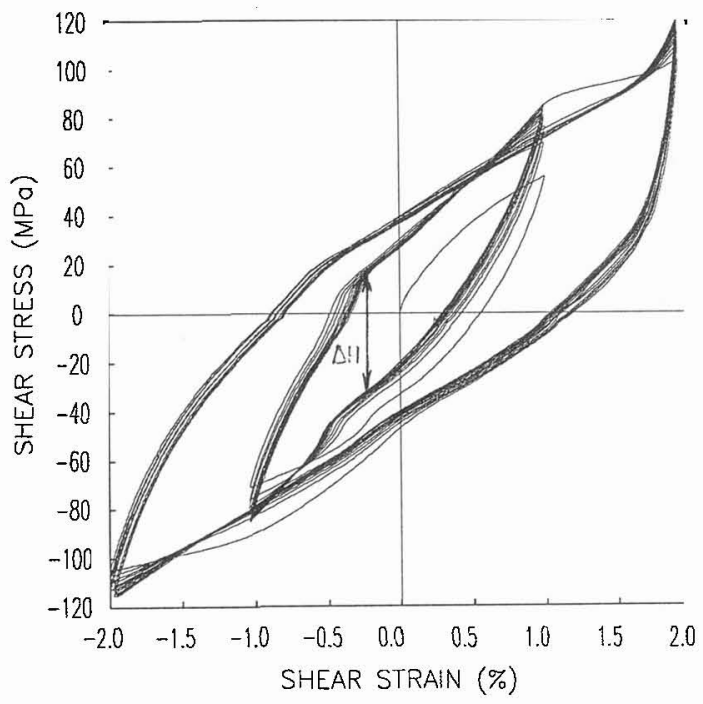

Fig.5 : Centred cyclic shear test

Conversely, the hysteresis decreases with the cycles, i.e, the difference $\Delta \mathrm{H}$ between the loading and unloading curves decreases [2], [3].

\section{IV) IDENTIFICATION OF THE MODEL}

To describe theoretically the macroscopic behavior of shape memory alloys, we use a elastohysteresis tensorial scheme [4]. This model is based on two fondamental stress contributions : a reversible one and a hysteretic one. The total stress is therefore the sum of a reversible stress and a hysteretic one, analogous to the chemical and effective stresses of otsuka [5]. For the martensitic phase, the main contribution is the hysteretic stress, whereas it is the reversible contribution which describes well the austenitic phase.

A simple identification of the shear tests (Fig.6), using 8 parameters, shows a good agreement between model predictions and the different isothermal experimental results of $\mathrm{Cu}-\mathrm{Zn}-\mathrm{Al}$ shape memory alloy.

\section{v) CONCLUSION}

This study shows the behavior of $\mathrm{Cu}-\mathrm{Zn}-\mathrm{Al}$ shape memory alloys under two different mechanical solicitations : tensile and shear tests. We paid a particular attention to both the mechanical and thermal homogeneity of the tests as well as to the accuracy of the local deformation measurement. This accuracy was obtained with a new video system using a camera CCD. Under such conditions, we performed reproductible tensile and shear tests. We showed that the anisotropy 
in the sheet plane of these materials is small, i.e, less than a few percent. Moreover, cyclic shear tests are in good agreement with previously published results.

The classical stress equivalents analysis indicates that the best results are obtained using Tresca and Von Mises criterions respectively for martensitic and austenitic phases. Eventually, the predictions of the elastohysteresis model fit well the experimental results, which confirms the basic assumptions of this scheme. The next step of our work, in order to check the full validity of the model, is to perform shear and tensile tests under variable temperatures. Such tests are in the process of being achieved.
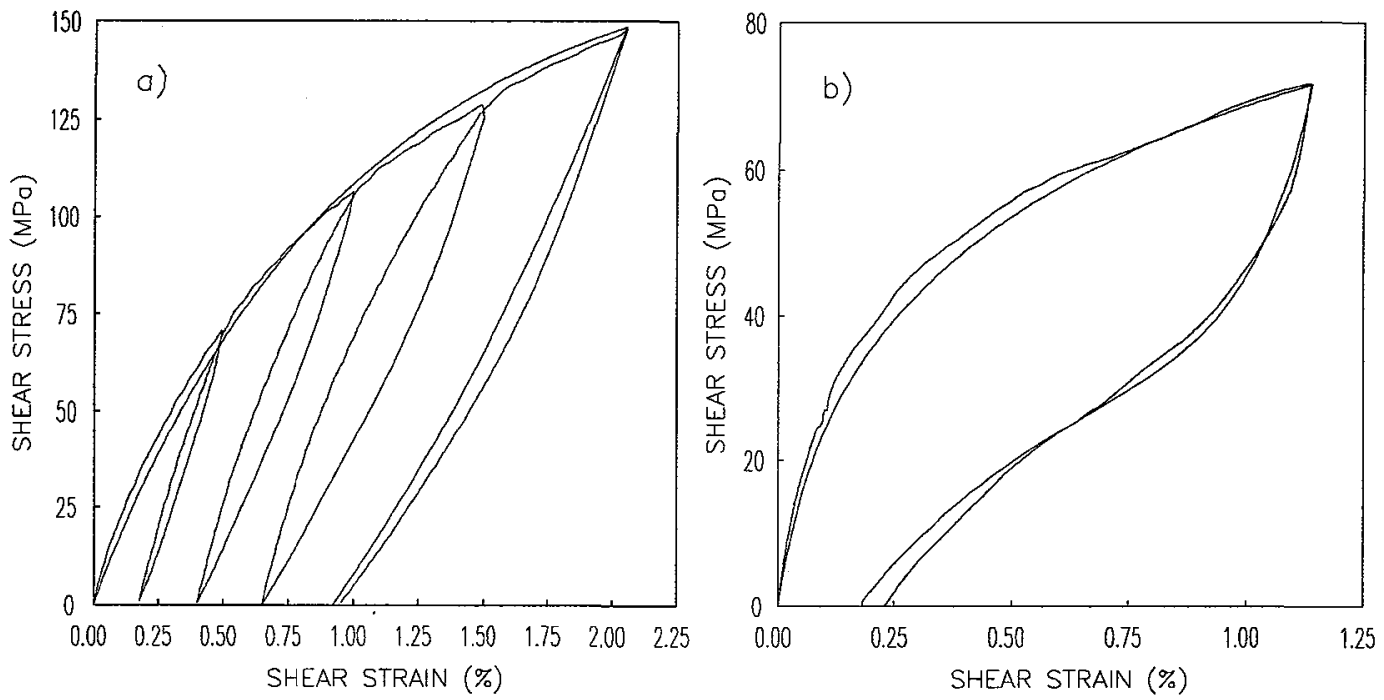

Fig.6 : identification of shear tests in the martensitic phase a) \& the austenitic phase b).

\section{Acknowledgement}

The authors are grateful to Professor Guénin and M.Morin of the Insa Lyon who supply us with the materials.

\section{References}

[1].E.Rauch, C.G'Sell : Mater. Sci. Eng., A111, 71, 1989.

[2].P.G.McCormick, Y.Liu : "Constitutive modelling of shape memory behavior", Proc. Int. Conference on constitutive laws for engineering materials, Chongging, China, pp 1-5, 1990.

[3].A.Tourabi, B.Wack, D.Favier : "Experimental determination of the hysteretic properties in shape memory alloys", Icomat 89, sydney, pp 535-540, 1989.

[4].D.Favier, P.Guélin, P.Pégon : "Thermomechanics of hysteresis effects in shape memory alloys", Icomat 89, Sydney, pp 559-564, 1989.
[5].K. Otsuka,
C.M.Wayman
K.Nakai.
H. Sakamoto,
K. Shimizu :

"Superelasticity effects and stress induced martensite transformations in Cu-Al-Ni alloys, Act. Met., 24, pp 207-226, 1976. 